\title{
THE PERIMETER OF OPTIMAL CONVEX LATTICE POLYGONS IN THE SENSE OF DIFFERENT METRICS
}

\author{
Miloš Stojaković
}

Classes of convex lattice polygons which have minimal $l_{p}$-perimeter with respect to the number of their vertices are said to be optimal in the sense of the $l_{p}$-metric.

It is proved that if $p$ and $q$ are arbitrary integers or $\infty$, the asymptotic expression for the $l_{q}$-perimeter of these optimal convex lattice polygons $Q_{p}(n)$ as a function of the number of their vertices $n$ is

$$
\operatorname{per}_{q}\left(Q_{p}(n)\right)=\frac{C_{p}^{q} \pi}{\sqrt{6 A_{p}^{3}}} n^{3 / 2}+O\left(n^{1+\varepsilon}\right) \text { for arbitrary } \varepsilon>0
$$

where

$$
C_{p}^{q}=\iint_{|x|^{p}+|y|^{p} \leqslant 1} \sqrt[q]{|x|^{q}+|y|^{q}} d x d y
$$

and $A_{p}$ is equal to the area of the planar shape $|x|^{p}+|y|^{p} \leqslant 1$.
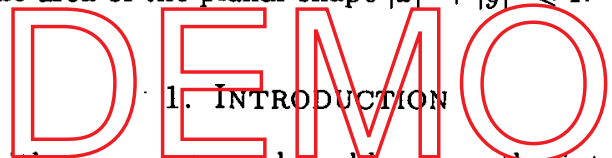

This paper deals with some extremal problems on the integer grid. Precisely, we shall consider some classes of optimal convex lattice polygons.

A convex lattice polygon is a polygon whose vertices are points on the integer lattice and whose interior angles are strictly less then $\pi$ radians (no three vertices are collinear). A convex lattice polygon with $n$ vertices is called an $n$-gon.

A convex lattice $n$-gon is said to be optimal in the sense of the $l_{p}$ metric if it has minimal $l_{p}$-perimeter with respect to the number of its vertices. Therefore, if a convex lattice $n$-gon has this property, its $l_{p}$-perimeter is equal to the minimum over all convex lattice $n$-gons $Q$ of the sum of the $l_{p}$-lengths of the edges of $Q$, and we denote it by $Q_{p}(n)$. The optimal polygon $Q_{p}(n)$ is not necessarily unique for every given integer $n$. Moreover, the explicit construction of a polygon $Q_{p}(2 k+1)$, where $p>1$ and $2 k+1$ is an arbitrary odd integer, is an open problem.

A classical paper of Jarnik [5] deals with $Q_{2}(n)$ polygons. He constructed a sequence of such polygons in order to solve the following problem: What is the maximal number of points from $\mathrm{Z}^{2}$ which lie on a continuous strictly convex curve $\gamma$ of length $s$, when

Received 7th August, 2000

Copyright Clearance Centre, Inc. Serial-fee code: 0004-9727/01 \$A2:00+0.00. 
$s$ tends to infinity? It turned out that this number is $(3 /(\sqrt[3]{2 \pi})) s^{2 / 3}+O\left(s^{1 / 3}\right)$. The exponent and constant in the leading term are the best possible. In [8] it is shown that the exponent $2 / 3$ can be decreased by imposing suitable smoothness condition on $\gamma$.

In particular, if $\gamma$ has a continuous third derivative with a sensible bound, the best possible value of the exponent lies between $3 / 5$ and $1 / 2$.

Since the function $f(x)=\sqrt{x}$ defined on $[0, n]$ is in $C^{\infty}([0, n])$ and the number of integral points on the curve $y=f(x)$ is $\left\lfloor n^{1 / 2}\right\rfloor$, obviously $1 / 2$ is the lower bound for the above-mentioned exponent.

In [7] it was proved that if $f \in C^{3}([0, n]),|f| \leqslant n$ and $f^{\prime \prime \prime} \neq 0$ on $[0, n]$, then the number of integral points on the curve $y=f(x)$ does not exceed $c(\varepsilon) \cdot n^{3 / 5+\varepsilon}$ for every $\varepsilon>0$, which gives the upper bound for the exponent.

Another class of optimal polygons is considered in [1]. The motivation for that research comes from digital geometry. The maximal number of vertices of a convex lattice polygon which can be inscribed into an $m \times m$ integer grid was considered. It was shown that this number is

Since the number of vertices of the convex of the set of $p$ oints is a parameter used for estimation of the time complexity in numerous algorithms, the previous result usually characterise the "worst case" situation on the squared integer grid of a given size.

The initial purpose of the research presented in this paper was to describe the asymptotic behaviour of the perimeter in the sense of the Euclidean metric, as well as the $l_{1}$ and $l_{\infty}$ metrics, of classes of optimal convex lattice polygons $Q_{p}(n)$ (where $n$ is an arbitrary integer). But since the theorems and the course of the proof in these cases can be generalised to an arbitrary $l_{q}$ metric (where $q$ is an integer, or $\infty$ ), all theorems are given in the general case. So it will be shown that the asymptotic expression for the $l_{q}$-perimeter of $l_{p}$-optimal polygons $Q_{p}(n)$ as a function of the number of its vertices, is

$$
\frac{C_{p}^{q} \pi}{\sqrt{6 A_{p}^{3}}} n^{3 / 2}+O\left(n^{1+\varepsilon}\right) \text { for arbitrary } \varepsilon>0
$$

where

$$
C_{p}^{q}=\iint_{|x|^{p+|y|^{p} \leqslant 1}} \sqrt[q]{|x|^{q}+|y|^{q}} d x d y
$$

and $A_{p}$ is equal to the area of the planar shape $|x|^{p}+|y|^{p} \leqslant 1$.

This result is derived for complete classes of optimal polygons even though their construction still remains unknown (including "Jarnik's polygons")-except in the case where $p=1$. (An exact construction of optimal $Q_{1}(n)$ polygons for arbitrary $n$ is given in $[6]$.) 
The case where $p=q=2$ is related to Jarnik's problem, while the case where $p=q=1$ is studied in $[1]$.

The choice of two different metrics for polygon optimality $\left(l_{p}\right)$ and measuring perimeter $\left(l_{q}\right)$ also gives some interesting results if $p \neq q$ (and especially if $p, q \in\{1,2, \infty\}$ ).

For example, if $p=1$ and $q=2$, we have the asymptotic expression for the Euclidean perimeter of convex lattice $n$-gons which have the minimal circumscribed square. This problem was studied in [10].

If $p=2$ and $q=1$ is taken, we get the solution to the problem equivalent to finding the asymptotic expression for the size of the circumscribed square of convex lattice $n$-gons with minimal Euclidian perimeter.

Other problems about convex lattice polygons were studied recently. In [4], the minimal area of convex lattice $n$-gons was considered. The explicit construction of convex lattice polygons (with $n$ vertices) whose area is $(15 / 784) n^{3}+o\left(n^{3}\right)((15 / 784) \approx 0.019133)$ was given.

As an application of the main result of this paper (an asymptotic expression for the Euclidean perimeter of $Q_{p}(n)$ ), an upper bound for the area of $Q_{p}(n)$ is derived. If $p=2$, that upper bound is $(1 / 54) n^{3}+O\left(n^{5 / 2+\varepsilon}\right)((1 / 54) \approx 0.018518)$, which improves the result from [4].

Some problems considering convex polyegns dettermined by lattice points on strictly convex curves containing the maximal number d l attice points, with respect to the length of the curve were studied in [3].

\section{Preliminaries}

If $a$ and $b$ are integers, $a \perp b$ means that the greatest common divisor for $a$ and $b$ is 1. Also, we shall say that $1 \perp 0$.

By $\mu(n)$ we shall denote the Möbius function, defined by

$$
\mu(1)=1 ;
$$

if $n>1$ and $n=p_{1}^{a_{1}} \cdots p_{k}^{a_{k}}$ is the prime decomposition of $n$, then

$$
\mu(n)=\left\{\begin{array}{cl}
(-1)^{k}, & \text { if } a_{1}=\ldots=a_{k}=1 \\
0, & \text { otherwise }
\end{array}\right.
$$

Let $e=\left(\left(x_{1}, y_{1}\right),\left(x_{2}, y_{2}\right)\right)$ be an edge of a convex lattice polygon. The $l_{p}$-distance length of $e$ is defined as

$$
l_{p}(e)=\sqrt[p]{\left|x_{2}-x_{1}\right|^{p}+\left|y_{2}-y_{1}\right|^{p}}, \quad p \geqslant 1 .
$$

We shall denote the differences $\left|x_{2}-x_{1}\right|$ and $\left|y_{2}-y_{1}\right|$ by $x(e)$ and $y(e)$, respectively. The slope of $e$ is defined to be quotient of these differences $y(e) / x(e)$. 
The perimeter in sense of the $l_{p}$ metric of a convex lattice polygon $Q$ is defined by

$$
\operatorname{per}_{p}(Q)=\sum_{e \text { is edge of } Q} l_{p}(e) .
$$

The $l_{q}$-perimeter of the convex lattice polygon $Q_{p}(n)$ will be denoted by $\operatorname{per}_{q}(n)$.

For $n>1, U_{p}(n)$ represents the partition function which counts the number of positive solutions of the equation $n=x^{p}+y^{p}$, where $x$ and $y$ are relatively prime integers. If $n=1$, we define $U_{p}(1)=1$ for $p=1,2, \ldots, \infty$ (we take $x=1, y=0$ as a solution).

In [9], the following sequence of integers was introduced:

$$
n_{p}(t)=4 \sum_{i=1}^{t} U_{p}(i), \quad t=1,2,3, \ldots
$$

First, we shall consider optimal lattice polygons with $n_{p}(t)(t=1,2, \ldots)$ vertices. It was shown in [9] by explicit construction that the optimal convex lattice polygon $Q_{p}\left(n_{p}(t)\right)$ is determined uniquely. For each integer $t, Q_{p}\left(n_{p}(t)\right)$ is constructed as follows, using a "greedy algorithm".

The polygon consists of four jsometric arcs, whose edge slopes coincide with the set

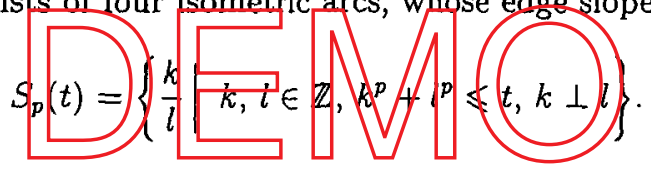

We shall denote the vertices of $Q_{p}\left(n_{p}(t)\right)$ in counterclockwise order by

$$
A_{0}=\left(x_{0}, y_{0}\right), \quad A_{1}=\left(x_{1}, y_{1}\right), \ldots, A_{n}=\left(x_{n_{p}(t)}, y_{n_{p}(t)}\right)=A_{0} .
$$

Let $e_{1}, e_{2}, \ldots e_{n_{p}(t)}$ be the edges determined by consecutive points from the previous sequence, that is,

$$
e_{1}=A_{0} A_{1}, \quad e_{2}=A_{1} A_{2}, \ldots, e_{n_{p}(t)}=A_{n_{p}(t)-1} A_{n_{p}(t)} .
$$

Then, the edges $e_{1}, e_{2}, \ldots, e_{n_{p}(t)}$ can be arranged into four arcs. If the angle between the positively oriented $x$-axis and the edge $A_{i-1} A_{i}$ is observed, then the south-east arc contains only the edges whose angles belong to $[0,(\pi / 2))$, the north-east arc contains only the edges whose angles belong to $[(\pi / 2), \pi)$, the north-west arc contains only the edges whose angles belong to $[\pi,(3 \pi / 2))$, and the south-west arc contains only the edges whose angles belong to $[(3 \pi / 2), 2 \pi)$.

The vertex $A_{0}$ is chosen to be one of the vertices, having the minimal $y$-coordinate, which has the minimal $x$-coordinate (the "lowest left" point), and then the vertex $A_{n_{p}(t) / 4}$ will be the one of the vertices having the maximal $x$-coordinate, which has the minimal $y$-coordinate (the "lowest outermost right" point). For convenience and without loss of 
generality, let us assume $A_{0}=(0,0)$. Since the slope of the edge $e_{i}$ is equal to $y\left(e_{i}\right) / x\left(e_{i}\right)$ it follows that the vertices of the south-east arc of the polygon $Q_{p}\left(n_{p}(t)\right)$ are:

$$
\begin{aligned}
A_{0}= & (0,0), \\
A_{1}= & \left(x\left(e_{1}\right), y\left(e_{1}\right)\right), \\
A_{2}= & \left(x\left(e_{1}\right)+x\left(e_{2}\right), y\left(e_{1}\right)+y\left(e_{2}\right)\right) \\
& \ldots \ldots \ldots \ldots \ldots \ldots \ldots \ldots \\
A_{n_{p}(t) / 4}= & \left(x\left(e_{1}\right)+x\left(e_{2}\right)+\ldots+x\left(e_{n_{p}(t) / 4}\right), y\left(e_{1}\right)+y\left(e_{2}\right)+\ldots+y\left(e_{n_{p}(t) / 4}\right)\right) .
\end{aligned}
$$

The slopes belonging to the south-east arc have to be arranged in increasing order

$$
\frac{0}{1}=\frac{y\left(e_{1}\right)}{x\left(e_{1}\right)}<\frac{y\left(e_{2}\right)}{x\left(e_{2}\right)}<\ldots<\frac{y\left(e_{n_{p}(t) / 4}\right)}{x\left(e_{n_{p}(t) / 4}\right)}
$$

and

$$
S_{p}(t)=\left\{\frac{y\left(e_{1}\right)}{x\left(e_{1}\right)}, \frac{y\left(e_{2}\right)}{x\left(e_{2}\right)}, \ldots, \frac{y\left(e_{n_{p}(t) / 4}\right)}{x\left(e_{n_{p}(t) / 4}\right)}\right\} .
$$

The remaining three arcs are btained the rotations oy $(\pi / 2), \pi$ and $(3 \pi / 2)$ ra-

It was proved in [9] that polygon constructed in his way is the unique convex lattice polygon with $n_{p}(t)$ vertices whose $l_{p}$-perimeter is minimal.

Thus, we have a sequence of integers representing the numbers of vertices of optimal convex lattice polygons (in sense of the $l_{p}$ metric) that can be explicitly constructed.

The following theorem gives the asymptotic expression for $n_{p}(t)$.

THEOREM 1. [9] The function $n_{p}(t)$ can be estimated by

$$
n_{p}(t)=\frac{6 A_{p}}{\pi^{2}} t^{2 / p}+O\left(t^{1 / p}\right)
$$

where $A_{p}$ equals the area of the planar shape $|x|^{p}+|y|^{p} \leqslant 1$.

A similar method is used to construct $Q_{p}(2 k)$, where $2 k$ is an even integer.

For every even integer $2 k$, there exists an integer $t$ such that $n(t-1) \leqslant 2 k<n(t)$. The polygon $Q_{p}(2 k)$ is constructed by adding edges to $Q_{p}(n(t-1))$. More precisely, $(2 k-n(t-1)) / 2$ edges of length $\sqrt[p]{t}$ are added to the south-east arc of $Q_{p}(n(t-1))$, and $(2 k-n(t-1)) / 2$ edges with the same slopes are added to the north-west arc of $Q_{p}(n(t-1))$, that is, for each edge $e$ added to the south-east arc, there is an edge $e^{\prime}$ added to the north-west arc such that $y\left(e^{\prime}\right) / x\left(e^{\prime}\right)=y(e) / x(e)\left(x(e) \perp y(e)\right.$ and $x\left(e^{\prime}\right) \perp y\left(e^{\prime}\right)$ are satisfied). Now it is easy to check that the $2 k$-gon obtained by this construction is optimal in sense of the $l_{p}$ metric. 
The explicit construction of $Q_{p}(2 k+1)$, where $2 k+1$ is an arbitrary odd integer, is an open problem for all $p>1$.

Each polygon $Q_{p}(n)$ has no more than 4 edges with the same slope, and this gives the following lower bound for the $l_{p}$-perimeter of $Q_{p}(n)$. If $n_{p}(t-1) \leqslant n<n_{p}(t)$, then

$$
\left(n-n_{p}(t-1)\right) \sqrt[p]{t}+4 \sum_{i=1}^{t-1} \sqrt[p]{i} U_{p}(i) \leqslant \operatorname{per}_{p}(n)
$$

Since this lower bound is established in a "greedy" manner, it will be called the greedy lower bound, and denoted by $\mathrm{glb}_{p}(n)$.

\section{THE $l_{q}$-PERIMETER OF $Q_{p}(n)$}

Let $P(v)$ be the number of lattice points $(a, b, c)$ different from the origin satisfying $a \perp b$, which belong to the 3-dimensional body

$$
D(v)=\left\{\left.(x, y, z)|| x\right|^{p}+|y|^{p} \leqslant v, 0<z \leqslant \sqrt[q]{|x|^{q}+|y|^{q}} \cdot\left(\frac{t}{v}\right)^{1 / p}\right\}
$$

and let $T(v)$ be the number of latice points $(a, p, c)$ different from the origin, which belong to $D(v)(a \perp b$ not required where ov is any positive number.

Now we consider the case $y=t$ The ognditior $|x|+|y| \leqslant t$ implies that $|x| \leqslant t^{1 / p}$ and $|y| \leqslant t^{1 / p}$. That means that the area of $D(t)$ is $O\left(t^{2 / p}\right)$. Therefore, we have that the $l_{q}$-perimeter of the optimal convex lattice polygon $Q_{p}\left(n_{p}(t)\right)$ is equal to $P(t)$ within an $O\left(t^{2 / p}\right)$ error, that is,

$$
\operatorname{per}_{q}\left(n_{p}(t)\right)=P(t)+O\left(t^{2 / p}\right) .
$$

The following lemma gives an asymptotic expression for $T(v)$, for arbitrary integers $p, q$.

LEMma 1. The following asymptotic equality holds:

$$
T(v)=C_{p}^{q} t^{1 / p} v^{2 / p}+O\left((v t)^{1 / p}\right)
$$

where

$$
C_{p}^{q}=\iint_{|x|^{p}+|y|^{p} \leqslant 1} \sqrt[q]{|x|^{q}+|y|^{q}} d x d y
$$

Proof: From the definition of $T(v)$, we have

$$
T(v)=\operatorname{volume}(D(v))+O(\operatorname{area}(D(v)))
$$


Thus,

$$
\begin{aligned}
T(v) & =\iint_{|x|^{p}+|y|^{p} \leqslant v}\left(\frac{t}{v}\right)^{1 / p} \sqrt[q]{|x|^{q}+|y|^{q}} d x d y+O\left((v t)^{1 / p}\right) \\
& =\iint_{|x|^{p}+|y|^{p} \leqslant 1} t^{1 / p} v^{2 / p} \sqrt[q]{|x|^{q}+|y|^{q}} d x d y+O\left((v t)^{1 / p}\right) \\
& =t^{1 / p} v^{2 / p} \iint_{|x|^{p}+|y|^{p} \leqslant 1} \sqrt[q]{|x|^{q}+|y|^{q}} d x d y+O\left((v t)^{1 / p}\right) \\
& =C_{p}^{q} t^{1 / p} v^{2 / p}+O\left((v t)^{1 / p}\right) .
\end{aligned}
$$

Now we can derive an asymptotic expression for the $l_{q}$-perimeter of the optimal lattice polygons $Q_{p}\left(n_{p}(t)\right)$, for arbitrary choice of $p$ and $q$.

THEOREM 2. The $l_{q}$-perimeter of the optimal convex lattice polygons $Q_{p}\left(n_{p}(t)\right)$ can be expressed as

$$
\operatorname{per}_{q}\left(n_{p}(t)\right)=\frac{6 C_{p}^{q}}{\pi^{2}} t^{3 / p}+O\left(t^{2 / p} \cdot \log t\right) .
$$

Proof: Obviously, the following equalities hold:

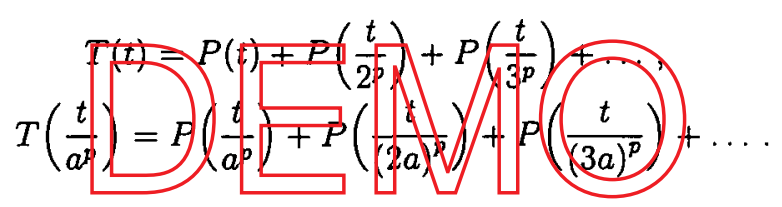

In the proof we shall use Lemma 1 , as well as the two following well-known equalities (see [2]):

$$
\begin{aligned}
& \sum_{a \mid l} \mu(a)= \begin{cases}1, & l=1 \\
0, & l>1\end{cases} \\
& \sum_{n=1}^{\infty} \frac{\mu(n)}{n^{2}}=\frac{1}{\zeta(2)}=\frac{6}{\pi^{2}}
\end{aligned}
$$

(where $\zeta$ denotes the Riemann zeta function).

Also, we shall use the inequality

$$
\left|\sum_{n=[\sqrt[P]{ }]+1}^{\infty} \frac{\mu(n)}{n^{2}}\right| \leqslant \sum_{n=[\mathbb{P} t]+1}^{\infty} \frac{1}{n^{2}}=O\left(\frac{1}{\sqrt[p]{t}}\right) .
$$

We have

$$
\begin{aligned}
P(t) & =\sum_{l=1}^{\infty} P\left(\frac{t}{l^{p}}\right)\left(\sum_{a \mid l} \mu(a)\right) \\
& =\sum_{n=1}^{[P / t]} \mu(n)\left(\sum_{m=1}^{\infty} P\left(\frac{t}{n^{p} m^{p}}\right)\right)
\end{aligned}
$$




$$
\begin{aligned}
& =\sum_{n=1}^{[\mathbb{E}]} \mu(n) T\left(\frac{t}{n^{p}}\right) \\
& =\sum_{n=1}^{[\sqrt[P]{t}]} \mu(n)\left(t^{1 / p}\left(\frac{t}{n^{p}}\right)^{2 / p} C_{p}^{q}+O\left(\left(\frac{t^{2}}{n^{p}}\right)^{1 / p}\right)\right) \\
& =\sum_{n=1}^{[P \sqrt{t}]} \mu(n) \frac{t^{3 / p}}{n^{2}} C_{p}^{q}+O\left(\sum_{n=1}^{\mid P[t]}|\mu(n)| \frac{t^{2 / p}}{n}\right) \\
& =C_{p}^{q} t^{3 / p}\left(\sum_{n=1}^{\infty} \frac{\mu(n)}{n^{2}}-\sum_{n=[p \sqrt{t}]+1}^{\infty} \frac{\mu(n)}{n^{2}}\right)+O\left(t^{2 / p} \cdot \log t\right) \\
& =C_{p}^{q} t^{3 / p} \frac{6}{\pi^{2}}+O\left(t^{2 / p} \cdot \log t\right) .
\end{aligned}
$$

On the other hand, from (1) we have that the $l_{q}$-perimeter of the optimal convex lattice polygon $Q_{p}\left(n_{p}(t)\right)$ is equal to $P(t)+O\left(t^{2 / p}\right)$, and the theorem is proved.

In order to derive the asymptotic expression for the $l_{q}$-perimeter of $Q_{p}(n)$ for an arbitrary integer $n$, we need the following lemma.

LEMMA 2. If $t$ is an integer such that

then the number of the edges of the polygon $Q p(2 / k)+1)($ longer then $\sqrt[p]{t}$ is bounded above by $O\left(t^{1 / p+\varepsilon}\right)$, for arbitraryes 0 .

Proof: Let $\gamma$ be the number of edges of $Q_{p}(2 k+1)$ longer then $\sqrt[p]{t}$. We shall prove that the assumption $\gamma \gg t^{1 / p+\varepsilon}$ leads to a contradiction.

So, assume that $\gamma \gg t^{1 / p+\varepsilon}$, for some $\varepsilon>0$. Since $2 k+1-n_{p}(t-1)<n_{p}(t)-$ $n_{p}(t-1)$ and $n_{p}(t)-n_{p}(t-1)=O\left(t^{1 / p}\right)$, we can determine the integer $\beta$ such that

$$
(2 k+1-n(t-1))+4 \sum_{s=t-\beta}^{t-1} U_{p}(s) \leqslant \gamma<(2 k+1-n(t-1))+4 \sum_{s=t-\beta-1}^{t-1} U_{p}(s) .
$$

Using the last inequality and Theorem 1 we derive the asymptotic expression for $\gamma$.

$$
\begin{aligned}
\gamma & =n_{p}(t)-n_{p}(t-\beta)+O\left(t^{1 / p}\right) \\
& =\frac{6 A_{p}}{\pi^{2}}\left(t^{2 / p}-(t-\beta)^{2 / p}\right)+O\left(t^{1 / p}\right) \\
& =\frac{6 A_{p}}{\pi^{2}} t^{2 / p}\left(1-\left(1-\frac{2}{p} \frac{\beta}{t}+O\left(\frac{\beta^{2}}{t^{2}}\right)\right)\right)+O\left(t^{1 / p}\right) \\
& =\frac{6 A_{p}}{\pi^{2}} \frac{2}{p} \frac{\beta}{t^{1-2 / p}}+O\left(\frac{\beta^{2}}{t^{2-2 / p}}\right)+O\left(t^{1 / p}\right) .
\end{aligned}
$$

Therefore, since $\gamma \gg t^{1 / p+\varepsilon}$, we have that $\beta \gg t^{1-1 / p+\varepsilon}$.

A lower bound for the $l_{p}$-perimeter of the convex lattice polygon $Q_{p}(2 k+1)$ can be derived if the following substitutions are made in $\operatorname{glb}_{p}(2 k+1)$ : 
- $4 \cdot U(t-1)$ summands equal to $\sqrt{t-1}$ are replaced by $\sqrt{t}$;

- $4 \cdot U(t-2)$ summands equal to $\sqrt{t-2}$ are replaced by $\sqrt{t}$;

- $4 \cdot U(t-\beta)$ summands equal to $\sqrt{t-\beta}$ are replaced by $\sqrt{t}$.

We have

$$
\operatorname{per}_{p}(2 k+1) \geqslant \operatorname{glb}_{p}(2 k+1)+4 \sum_{s=t-\beta}^{t}(\sqrt[p]{t}-\sqrt[p]{s}) U_{p}(s) .
$$

First, we shall prove the following asymptotic equation.

$$
4 \sum_{s=t-\beta}^{t}(t-s) U_{p}(s)=\frac{12 A_{p}}{\pi^{2}} \beta t^{2 / p}+O\left(\frac{\beta^{2}}{t^{1-2 / p}}\right)+O\left(t^{1 / p} \beta\right)
$$

In the proof, Stieltjes integration is used.

$$
\begin{aligned}
& 4 \sum_{s=t-\beta}^{t}(t-s) U_{p}(s)=\int_{t-\beta}^{t}(t-s) d\left(n_{p}(s)\right)
\end{aligned}
$$

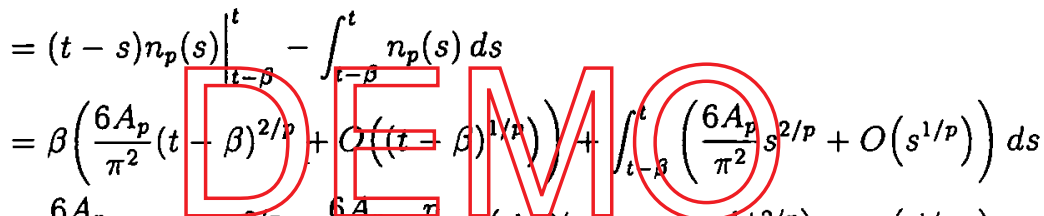

$$
\begin{aligned}
& =\frac{6 A_{p}}{\pi^{2}} \beta(t-\beta)^{2 / p}+\frac{6 A_{p}}{\pi^{2}} \frac{p}{p+2}\left(t^{1+2 / p}-(t-\beta)^{1+2 / p}\right)+O\left(t^{1 / p} \beta\right) \\
& =\frac{6 A_{p}}{\pi^{2}}\left(\beta t^{2 / p}\left(1-\frac{2}{p} \frac{\beta}{t}+O\left(\frac{\beta^{2}}{t^{2}}\right)\right)\right. \\
& \left.+\frac{p}{p+2} t^{1+2 / p}\left(\left(1+\frac{2}{p}\right) \frac{\beta}{t}+O\left(\frac{\beta^{2}}{t^{2}}\right)\right)\right)+O\left(t^{1 / p} \beta\right) \\
& =\frac{6 A_{p}}{\pi^{2}}\left(2 \beta t^{2 / p}-\frac{2}{p} \frac{\beta^{2}}{t^{1-2 / p}}+O\left(\frac{\beta^{2}}{t^{1-2 / p}}\right)\right)+O\left(t^{1 / p} \beta\right) \\
& =\frac{12 A_{p}}{\pi^{2}} \beta t^{2 / p}+O\left(\frac{\beta^{2}}{t^{1-2 / p}}\right)+O\left(t^{1 / p} \beta\right) \text {. }
\end{aligned}
$$

Now, using (2) we have

$$
\begin{aligned}
4 \sum_{s=t-\beta}^{t}(\sqrt[p]{t}-\sqrt[p]{s}) U_{p}(s) & =4 \sum_{s=t-\beta}^{t} \frac{t-s}{t^{1-1 / p}+t^{1-2 / p} s^{1 / p}+\ldots+s^{1-1 / p}} U_{p}(s) \\
& \geqslant \frac{1}{p t^{1-1 / p}} 4 \sum_{s=t-\beta}^{t}(t-s) U_{p}(s) \\
& =\frac{1}{p t^{1-1 / p}}\left(\frac{12 A_{p}}{\pi^{2}} \beta t^{2 / p}+O\left(\frac{\beta^{2}}{t^{1-2 / p}}\right)+O\left(t^{1 / p} \beta\right)\right)
\end{aligned}
$$




$$
=\frac{12 A_{p}}{p \pi^{2}} \frac{\beta}{t^{1-3 / p}}+O\left(\frac{\beta^{2}}{t^{2-3 / p}}\right)+O\left(\frac{\beta}{t^{1-2 / p}}\right) .
$$

Since $\beta \gg t^{1-1 / p+\varepsilon}$, we can finally obtain the asymptotic lower bound for the $l_{p^{-}}$ perimeter of the convex lattice polygon $Q_{p}(2 k+1)$,

$$
\operatorname{per}_{p}(2 k+1) \gg \operatorname{glb}_{p}(2 k+1)+\frac{12 A_{p}}{p \pi^{2}} t^{2 / p+\varepsilon} .
$$

But on the other hand, the way of constructing the polygon $Q_{p}(2 k+2)$ implies that

$$
\operatorname{per}_{p}(2 k+1)<\operatorname{glb}_{p}(2 k+1)+t^{1 / p}=\operatorname{glb}_{p}(2 k+2)=\operatorname{per}_{p}(2 k+2) .
$$

The last two inequalities give a contradiction.

Therefore, we have that $\gamma=O\left(t^{1 / p+\varepsilon}\right)$.

THEOREM 3. If $t$ is an integer such that $n_{p}(t-1) \leqslant n<n_{p}(t)$, then the asymptotic expression for the $l_{q}$-perimeter of the optimal convex lattice polygon $Q_{p}(n)$ as a function of $t$, is

$$
\operatorname{per}_{q}(n)=\frac{6 C_{p}^{q}}{\pi^{2}} t^{3 / p}+O\left(t^{2 / p+\varepsilon}\right) .
$$

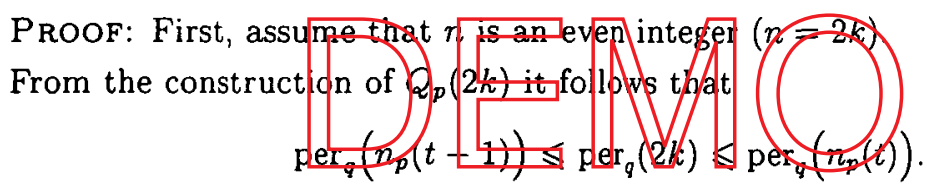

From Theorem 2 we have that

$$
\operatorname{per}_{q}(2 k)=\frac{6 C_{p}^{q}}{\pi^{2}} t^{3 / p}+O\left(t^{2 / p+\varepsilon}\right) .
$$

The error estimate $O\left(t^{2 / p+\varepsilon}\right)$ in the last equation is obviously not the best possible in this case (that $n$ is an even integer), but it is satisfactory since it equals the best possible error in the next case.

Now, assume that $n$ is an odd integer $(n=2 k+1)$.

First we shall prove that the lengths of the edges of the polygon $Q_{p}(2 k+1)$ are bounded above. If we assume that there is an edge of $Q_{p}(2 k+1)$ longer (in the sense of the $l_{p}$ metric) than $2 \sqrt[p]{t}$, then we have

$$
\operatorname{per}_{p}(2 k+1)>\operatorname{glb}_{p}(2 k+1)+\sqrt[p]{t}=\operatorname{glb}_{p}(2 k+2)=\operatorname{per}_{p}(2 k+2),
$$

which is a contradiction.

Thus, there is no edge of $Q_{p}(2 k+1)$ longer then $2 \sqrt[p]{t}$. We can derive an upper bound for the length of an edge of $Q_{p}(2 k+1)$ in the sense of the $l_{q}$ metric: $l_{p}(e)=$ $\sqrt[p]{|x(e)|^{p}+|y(e)|^{p}} \leqslant 2 \sqrt[p]{t}$ so $|x|^{p} \leqslant 2^{p} t$, and $|y|^{p} \leqslant 2^{p} t$ and hence

$$
l_{q}(e)=\sqrt[q]{|x(e)|^{q}+|y(e)|^{q}} \leqslant 2 \sqrt[q]{2} \cdot \sqrt[p]{t} .
$$


Finally, we have

$$
\begin{aligned}
\operatorname{per}_{q}\left(Q_{p}(n(t))\right)-\operatorname{per}_{q}\left(Q_{p}(2 k+1)\right) \mid & \leqslant \max (2 \sqrt[q]{2} \cdot \sqrt[p]{t} \cdot \gamma, \sqrt[q]{2} \cdot \sqrt[p]{t} \cdot(n(t)-(2 k+1))) \\
& =\max \left(2 \sqrt[q]{2} \cdot \sqrt[p]{t} \cdot O\left(t^{1 / p+\varepsilon}\right), \sqrt[q]{2} \cdot \sqrt[p]{t} \cdot O\left(t^{1 / p}\right)\right) \\
& =O\left(t^{2 / p+\varepsilon}\right),
\end{aligned}
$$

and

$$
\operatorname{per}_{q}(2 k+1)=\operatorname{per}_{q}(n(t))+O\left(t^{2 / p+\varepsilon}\right)=\frac{6 C_{p}^{q}}{\pi^{2}} t^{3 / p}+O\left(t^{2 / p+\varepsilon}\right) .
$$

Now we can give the asymptotic expression for the $l_{q}$-perimeter of all the optimal $n$-gons as a function of $n$.

THEOREM 4. The $l_{q}$-perimeter of the optimal convex lattice polygon $Q_{p}(n)$ expressed as a function of the number of its vertices $n$, is

$$
\operatorname{per}_{q}(n)=\frac{C_{p}^{q} \pi}{\sqrt{6 A_{p}^{3}}} n^{3 / 2}+O\left(n^{1+\varepsilon}\right)
$$

for arbitrary $\varepsilon>0$, and $p, q \in\{1,2, \ldots, \infty\}$.

PROOF: For every integer op, there is an integer $t$ sych that

From the last inequality and Theorem 1 we have that the asymptotic expression for $n$ is

$$
n=\frac{6 A_{p}}{\pi^{2}} t^{2 / p}+O\left(t^{1 / p}\right) \text {. }
$$

Since $t=O\left(n^{p / 2}\right)$, we obtain the asymptotic expression for $t$ as a function of $n$ :

$$
t=\frac{\pi^{p}}{\sqrt{\left(6 A_{p}\right)^{p}}} n^{p / 2}+O\left(n^{(p-1) / 2}\right)
$$

From Theorem 3 we have that

$$
\operatorname{per}_{q}(n)=\frac{6 C_{p}^{q}}{\pi^{2}} t^{3 / p}+O\left(t^{2 / p+\varepsilon}\right) .
$$

Thus, by eliminating the variable $t$ in the expression representing $\operatorname{per}_{q}(n)$ we have

$$
\begin{aligned}
\operatorname{per}_{q}(n) & =\frac{6 C_{p}^{q}}{\pi^{2}}\left(\frac{\pi^{p}}{\sqrt{\left(6 A_{p}\right)^{p}}} n^{p / 2}+O\left(n^{(p-1) / 2}\right)\right)^{3 / p}+O\left(n^{1+\bar{\varepsilon}}\right) \\
& =\frac{6 C_{p}^{q} \pi}{\left(6 A_{p}\right)^{3 / 2}} n^{3 / 2}\left(1+O\left(n^{-1 / 2}\right)\right)^{3 / p}+O\left(n^{1+\bar{\varepsilon}}\right) \\
& =\frac{C_{p}^{q} \pi}{\sqrt{6 A_{p}^{3}}} n^{3 / 2}+O\left(n^{1+\bar{\varepsilon}}\right),
\end{aligned}
$$


where $\tilde{\varepsilon}=\varepsilon p / 2$.

The next corollary is an application of the result presented in Theorem 4 giving the asymptotic upper bound for the area of the optimal convex lattice polygons $Q_{p}(n)$.

COROLLARY 1. The following asymptotic inequality holds:

$$
P\left(Q_{p}(n)\right) \leqslant B_{p} n^{3}+O\left(n^{5 / 2+\varepsilon}\right)
$$

where

$$
B_{p}=\frac{\left(C_{p}^{2}\right)^{2} \pi}{24 A_{p}^{3}}
$$

and $P(Q)$ denotes the area of the planar shape $Q$.

Proof: It is known that of all planar shapes with fixed perimeter the circle has the greatest area. Since we have an asymptotic expression for the Euclidean perimeter of $Q_{p}(n)$ as a function of $n$ (Theorem $4, q=2$ ) the statement of the theorem follows directly.

In the case that $p=2$ we have $B_{2}=1 / 54 \approx 0.018518$. In the case that $p \in\{1, \infty\}$ we have

Note. If we define

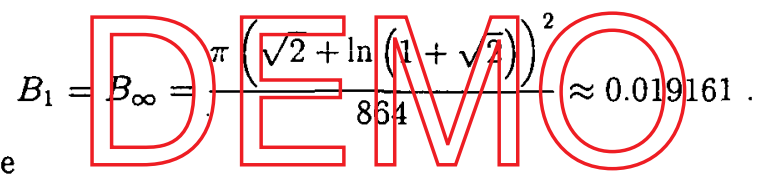

$$
D(v)=\left\{(x, y, z)|| x|\leqslant v,| y \mid \leqslant v, 0<z \leqslant \sqrt[q]{|x|^{q}+|y|^{q}} \cdot \frac{t}{v}\right\},
$$

then using a slight modification of the proofs of Lemma 1 and Theorem 2, we get that the asymptotic expression for the $l_{q}$-perimeter of the optimal convex lattice polygon $Q_{\infty}\left(n_{\infty}(t)\right)(p=\infty)$ is

$$
\operatorname{per}_{q}\left(n_{\infty}(t)\right)=\frac{6 C_{\infty}^{q}}{\pi^{2}} t^{3}+O\left(t^{2} \cdot \log t\right)
$$

where

$$
C_{\infty}^{q}=\iint_{|x| \leqslant 1,|y| \leqslant 1} \sqrt[q]{|x|^{q}+|y|^{q}} d x d y .
$$

Also, in the case that $q=\infty$ the proof gives the following coefficient

$$
C_{p}^{\infty}=\iint_{|x|^{p}+|y|^{p} \leqslant 1} \max \{|x|,|y|\} d x d y .
$$

In both previous cases we have

$$
\begin{aligned}
& C_{\infty}^{q}=\lim _{p \rightarrow \infty} C_{p}^{q}=\lim _{p \rightarrow \infty} \iint_{|x|^{p}+|y|^{p} \leqslant 1} \sqrt[q]{|x|^{q}+|y|^{q}} d x d y, \\
& C_{p}^{\infty}=\lim _{q \rightarrow \infty} C_{p}^{q}=\lim _{q \rightarrow \infty} \iint_{|x|^{p}+|y|^{p} \leqslant 1}^{q} \sqrt[q]{|x|^{q}+|y|^{q}} d x d y .
\end{aligned}
$$


In the case that $p=q=\infty$, we have

$$
C_{\infty}^{\infty}=\iint_{|x| \leqslant 1,|y| \leqslant 1} \max \{|x|,|y|\} d x d y=\frac{8}{3}
$$

If we denote the coefficient $\left(C_{p}^{q} \pi\right) /\left(\sqrt{6 A_{p}^{3}}\right)$ in the leading term from Theorem 4 by $K_{p}^{q}$, we have the following exact values:

$$
\begin{array}{lll}
K_{1}^{1}=\frac{\pi}{3 \sqrt{3}}, & K_{1}^{2}=\frac{\pi(2+\sqrt{2} \ln (1+\sqrt{2}))}{12 \sqrt{3}}, & K_{1}^{\infty}=\frac{\pi}{4 \sqrt{3}} \\
K_{2}^{1}=\frac{8}{3 \sqrt{6 \pi}}, & K_{2}^{2}=\frac{\sqrt{2 \pi}}{3 \sqrt{3}}, & K_{2}^{\infty}=\frac{4}{3 \sqrt{3 \pi}} \\
K_{\infty}^{1}=\frac{\pi}{2 \sqrt{6}}, & K_{\infty}^{2}=\frac{\pi(2+\sqrt{2} \ln (1+\sqrt{2}))}{12 \sqrt{3}}, & K_{\infty}^{\infty}=\frac{\pi}{3 \sqrt{6}}
\end{array}
$$

\begin{tabular}{|c|c|c|c|c|c|}
\hline$p^{q}$ & 1 & 2 & 3 & 5 & $\infty$ \\
\hline 1 & 0.6046 & 0.4907 & 0.46957 & 0.45902 & 0.45345 \\
\hline 2 & 0.61421 & 0.4824 & 0.456 & 0.44207 & 0.43431 \\
\hline 3 & c. .52269 & c. 48359 & & 0,43931 & 0.43033 \\
\hline 5 & c. 63129 & c. 4863 & 564 & 0.43857 & 0.42835 \\
\hline$\infty$ & d.64122 & d. $\frac{4007}{2}$ & 813 & - & 0.42752 \\
\hline
\end{tabular}

Finally, we give some approximate values of $K_{p}^{q}$ :

The decrease of the coefficient value in a row (from left to right) represents the decrease of the $l_{q}$ metric as $q$ increases. The coefficient value in a column $q$ is the least for $p=q$ (diagonal value), because in that case the perimeter is measured by the same metric that is used for optimality.

\section{REFERENCES}

[1] D.M. Acketa and J. Žunić, 'On the maximal number of edges of convex digital polygons included into a $m \times m$-grid', J. Combin. Theory Ser. A 69 (1995), 358-368.

[2] T.M. Apostol, Introduction to analytic number theory (Springer-Verlag, Berlin, Heidelberg, New York, 1976).

[3] E. Bombieri and J. Pila, 'The number of integral points on arcs and ovals', Duke Math. J. 59 (1989), 337-357.

[4] J.C. Colburn and R.J. Simpson, 'A note on bounds on the minimum area of convex lattice polygons', Bull. Austral. Math. Soc. 45 (1992), 237-240.

[5] V. Jarnik, 'Über die Gitterpunkte auf konvexen Kurven', Math. Z. 24 (1925), 500-518.

[6] S. Matić-Kekić, D.M. Acketa and J. Žunić, 'An exact construction of digital convex polygons with minimal diameter', Discrete Math. 150 (1996), 303-313.

[7] W.M. Schmidt, 'Integer points on curves and surfaces', Monatsh. Math. 99 (1985), 45-72.

[8] H.P.F. Swinerton-Dyer, 'The number of lattice points on a convex curve', $J$. Number Theory 6 (1974), 128-135. 
[9] J. Żunić, 'Extremal problems on convex lattice polygons in sense of $l_{p}$-metrics', (private communication).

[10] J. Žunić, 'Notes on optimal convex lattice polygons', Bull. London Math. Soc. 30 (1998), 377-385.

Institute of Mathematics, Faculty of Science

University of Novi Sad

Trg D. Obradovića 4

21000 Novi Sad

Yugoslavia

e-mail: MilosSt@EUnet.yu

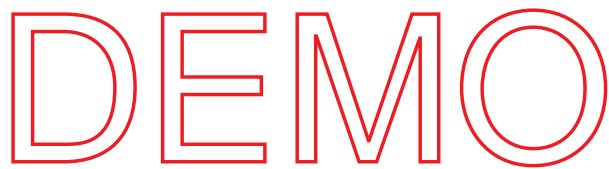

